Journal of Animal and Veterinary Advances 11 (15): 2828-2838, 2012

ISSN: $1680-5593$

(C) Medwell Journals, 2012

\title{
Comparative Immunomic Analysis of Schistosoma japonicum Soluble Egg Antigens Reacting with Patient Sera Before and after Praziquantel Treatment
}

\author{
${ }^{1,}{ }^{2} \mathrm{C} . \mathrm{Ju},{ }^{2} \mathrm{~B} . \mathrm{Xu},{ }^{2} \mathrm{Y}$. Lu, ${ }^{2} \mathrm{X}$.J. Mo, ${ }^{2}$ T. Zhang, ${ }^{2} \mathrm{~S}$.B Chen, ${ }^{3} \mathrm{~F}$. Liu, ${ }^{4}$ S. J. Cui, ${ }^{2}$ W. Liu, \\ ${ }^{2}$ J.H. Chen, ${ }^{2} \mathrm{Z}$. Feng, ${ }^{1} \mathrm{~J}$.X. Peng and ${ }^{2} \mathrm{~W}$. Hu \\ ${ }^{1}$ Institute of Entomology, School of Life Science, Central China Normal University, \\ 430010 Wuhan, P.R. China \\ ${ }^{2}$ Key Laboratory of Parasitology and Vector Biology, National Institute of Parasitic Diseases, \\ Chinese Center for Disease Control and Prevention, \\ WHO Collaborating Centre for Malaria, \\ Schistosomiasis and Filariasis, Shanghai, China \\ ${ }^{3}$ Institutes of Biomedical Sciences, Fudan University, Shanghai, China \\ ${ }^{4}$ Institute for Advanced Interdisciplinary Research, East China Normal University, Shanghai, China
}

\begin{abstract}
Schistosomiasis ranks as the second most serious parasitic disease worldwide after malaria. More than 250 million people are infected with schistosomes in the tropics or subtropics. The treatment and control of schistosomiasis which is a major neglected tropical parasitic disease, depends almost exclusively on chemotherapy with Praziquantel (PZQ). Current serologic diagnostic assays have shown that schistosome specific antibodies in human serum may remain for at least 1 year after cure. Repeated administration of PZQ for a long time might induce drug resistance to the parasite which is a big challenge for strategizing for the prevention and control of schistosomiasis. As schistosome eggs represent the most pathogenic form causing the disease, it is essential to determine if and how the level of antibodies against schistosome Soluble Egg Antigens (SEA) is affected by PZQ treatment. In this study, researchers carried out an immunomic analysis to profile Schistosoma japonicum SEA reacting with pooled human serum samples of pre and post treatment with PZQ by two dimensional electrophoresis combined with Western blotting. A total of 67 protein spots that were serologically recognized by serum samples were successfully subjected to mass spectrometric analysis. Of them, 37 different characterized proteins were successfully identified. Furthermore, of 67 protein spots, the reactivity of 49 protein spots to sera was reduced 20 weeks after PZQ treatment whereas only 5 spots showed increases in the intensity of recognition by post treatment sera. The present study suggested that chemotherapy with PZQ mainly affects the intensity of serological recognition of $S$. japonicum SEA. The immunomic proteins that were identified may facilitate a better understanding of the egg induced pathogenesis of schistosomiasis and host-parasite interplay and may provide potential targets for the diagnosis and evaluation of treatment for the disease as well.
\end{abstract}

$\underline{\text { Key words: Schistosoma japonicum, immunomic analysis, soluble egg antigens, praziquantel, protein, China }}$

\section{INTRODUCTION}

Schistosomiasis remains a major neglected tropical parasitic disease affecting $>200$ million people in 76 countries. About 120 million have symptoms and 20 million suffer severe illness (Chitsulo et al., 2000; Hotez et al., 2008). Currently due to the absence of vaccines, efficient vector controls and water sanitation, the treatment of schistosomiasis still depends almost exclusively on Praziquantel (PZQ) although several recent drug candidates have exhibited potential anti-schistosomal properties in the laboratory such as oxadiazoles (Sayed et al., 2008), cysteine protease inhibitor K11777 (Abdulla et al., 2007) and mefloquine (Keiser et al., 2009). PZQ was first synthesized in the 1970 s (Seubert et al., 1977) and subsequently introduced for the treatment of schistosomiasis. Owing to its safety, broad anthelmintic spectrum and reasonable cost, $>100$ million people have been treated with $\mathrm{PZQ}$ in schistosomiasis endemic areas (Cioli, 2000). Because PZQ targets the adult worm but has only minor activity against the young developing stages (i.e., schistosomula)

Corresponding Author: J.X. Peng, Institute of Entomology, Central China Normal University, 430010 Wuhan, P.R. China 
(Xiao et al., 1985, 1987), retreatment is recommended to kill the parasites (Renganathan and Cioli, 1998). However, its mechanism of action has yet to be fully elucidated (Greenberg, 2005).

Diagnosis as well as chemotherapy is central to the control of schistosomiasis. Although, stool examination is the gold standard for diagnosing or monitoring the response to treatment in schistosomiasis cases, it is labor intensive and time-consuming. In addition, the sensitivity of this method is poor in the endemic areas with low prevalence. Immunodiagnostic techniques are highly sensitive, easy to perform and are useful epidemiological tools for the screening of target populations in endemic areas. However, most immunodiagnostic assays currently applied in China have indicated that the anti-schistosome antibody level has remained high for at least 1 year after cure and in many cases much longer ( $\mathrm{Zhu}, 2005)$ which leads to the problem that a positive sero-test may be found to be fecal egg negative. Repeated administration of $\mathrm{PZQ}$ for a long time might induce drug resistance to the parasite (Doenhoff et al., 2002; Doenhoff and Mattoccia, 2006) which is a big challenge for strategizing for the prevention and control of schistosomiasis. Therefore, it is critical to identify the immunogenic antigens that could distinguish the cured cases from those with an ongoing infection.

Schistosome eggs are the primary cause of pathogenesis in schistosomiasis and egg antigens play a critical role in regulating host immune responses to the helminth (Pearce and MacDonald, 2002). In addition, there is evidence that treatment with $P Z Q$ could lead to changes in the Soluble Egg Antigen (SEA)-induced immune responses (Martins-Leite et al., 2008) and variations in specific anti-egg antibody levels which could reflect the current antigen levels (Mutapi et al., 1998). Also, the average number of eggs produced by Schistosoma japonicum per day is much more than other schistosome species (Moore and Sandground, 1956). Hence, knowledge of the effects of PZQ on the level of antibodies against $S$. japonicum SEAs is essential for evaluating chemotherapy efficacy which may also be a useful complementary way to carry out surveillance of schistosomiasis in epidemic areas (Wang et al., 2008). Furthermore, such knowledge is able to facilitate an understanding of egg induced pathology during schistosomiasis.

Crude antigens are valuable for detecting general patterns of immune responses in infected populations. However, single antigens permit a better definition of humoral and cellular responses under investigation (Mutapi, 2001). The advent of the omics era has yielded an avalanche of helminth omics data that provide valuable resources for defining those immunogenic proteins and investigating the intriguing biology of helminth parasites (Ju et al., 2010). A traditional expression library screening method has its limitations in the detection of those proteins with post translation modifications but a combination of 2-Dimensional Electrophoresis (2DE) and Western Blotting (2D-WB) can circumvent this problem and cover a wider range of antigenic targets (Wilson et al., 2004). A number of $S$. haematobium worm antigenic proteins recognized by patient serum samples before and after curative PZQ treatment were identified by 2DE, Western blotting and Mass Spectrometry (MS) (Mutapi et al., 2005). The combination of proteomic and serological techniques was subsequently used to analyze the components of immunogenic proteins from the tegument and excretory-secretory products of adult S. bovis (Perez-Sanchez et al., 2006). More recently, several proteomic investigations have addressed schistosomal egg-associated proteins including SEA (Curwen et al., 2004), Egg Secreted Proteins (ESP) (Cass et al., 2007) and the soluble constituents in developed and undeveloped eggs (Mathieson and Wilson, 2010). In addition, the identification of schistosome specific proteins will also profit from comprehensive genome analysis of the blood flukes S. japonicum (Liu et al., 2009b) and S. mansoni (Berriman et al., 2009) such as an investigation of vaccine candidate immunomics screening with $S$. mansoni protein microarrays (Driguez et al., 2010). To date, there have been no reports determining the effect of treatment with $\mathrm{PZQ}$ on S.japonicum egg immnunogenic antigen profiles, however such investigations would be valuable for a better understanding of the egg induced pathogenesis of schistosomiasis and host parasite interplays, provide potential targets for diagnosis and evaluation of treatment for the disease as well.

To identify and characterize the immunogenic proteins of S. japonicum, researchers combined 2DE and Western blot analysis to determine the proteins of SEA that were recognized by pooled serum samples of patients with $S$. japonicum before and after $\mathrm{PZQ}$ treatment and researchers compared the difference in serological recognition between them.

\section{MATERIALS AND METHODS}

Ethics statement: Ethical clearance was granted by the Ethics Committee of the National Institute of Parasitic Diseases, Chinese Center for Disease Control and Prevention in Shanghai, P.R. China (Ref No.: 20100525-1). The objectives, procedures and potential risks were orally explained to all participants. A signed written consent 
form was also obtained from each participant or their proxy. All experimental uses of animals for the studies described in this study were also approved by the Institutional Animal Care and Use Committee.

Serum samples: Human serum samples were obtained from the residents living in Miaoling village in Anhui province of China where $S$. japonicum is endemic. Diagnoses were confirmed by the improved Kato-Katz stool examination (Katz et al., 1972) in which two fresh stool samples collected from each subject on two consecutive days were used to prepare six slides, coupled with a hatching test (Kassim and Gibertson, 1976). Following the initial examinations, all infected residents who had never been treated for any helminth infection agreed to receive chemotherapy with $\mathrm{PZQ}$ at the standard dose of $40 \mathrm{mg} \mathrm{kg}^{-1}$ body weight. Serological samples were collected from the residents that were confirmed as egg negative 20 weeks after treatment. Of these, 10 residents that donated both pre and post treatment sera were selected for the study. The sex ratio (males vs. females) of these compliant participants (19-54 years old) was approximately 1.5. Sera from persons free of $S$. japonicum from non endemic areas were included as normal controls. Almost no cross reaction with Clonorchis sinensis and Fasciola buski were found in all the studied serum samples. The treatment was performed in the schistosomiasis non-transmission season to avoid re-infection (Table 1).

Preparation of soluble egg antigens: The Anhui strain of S.japonicum, maintained in Oncomelania hupensis snails at the National Institute of Parasitic Diseases (Shanghai, China) was used for the experiments. Schistosome eggs were collected from the livers of New Zealand white rabbits that had each been percutaneously exposed to 800 1000 S. japonicum cercariae 42 45 days earlier using a method to isolate schistosome eggs free of host cells and debris (Dalton et al., 1997). Eggs were ground in a mortar and then sonicated (Boros and Warren, 1970) 8 times for $5 \mathrm{sec}$ in lysis buffer (9.5 M urea, $65 \mathrm{mMDTT}$, $4 \%$ CHAPS and $0.2 \%$ IPG buffer) containing protease inhibitors, followed by centrifugation for $45 \mathrm{~min}$ at

Table 1: The information of sera samples in Miaoling village

\begin{tabular}{llccc}
\hline ID & Sex & Ages & EPG (pre-treatment) & EPG (post-treatment) \\
\hline 400801 & Male & 19 & 4 & 0 \\
806402 & Female & 35 & 16 & 0 \\
702401 & Male & 28 & 96 & 0 \\
703501 & Male & 47 & 8 & 0 \\
700401 & Male & 54 & 12 & 0 \\
702502 & Female & 33 & 28 & 0 \\
702501 & Female & 21 & 80 & 0 \\
703405 & Male & 36 & 8 & 0 \\
703401 & Male & 44 & 16 & 0 \\
702701 & Female & 29 & 4 & 0 \\
\hline
\end{tabular}

$12,000 \mathrm{~g}$ and $4^{\circ} \mathrm{C}$. The SEA were produced from the supernatant and protein concentrations were determined using the Bradford assay. The samples were then stored at $-80^{\circ} \mathrm{C}$ until needed.

2-Dimensional Electrophoresis (2-DE): 2-dimensional separation gels with SEA were performed in parallel; 100 and $800 \mu \mathrm{g}$ SEA were loaded onto analytical and preparative gels, respectively. Isoelectric Focusing (IEF) was performed in $13 \mathrm{~cm}$ IPG strips (Amersham) with a range of $\mathrm{pH} 3-\mathrm{pH} 10$. The IEF was accomplished using the Ettan IPGphor Isoelectric Focusing System (Amersham). The soluble antigens were mixed with $250 \mu \mathrm{L}$ rehydration solution containing $8 \mathrm{M}$ urea, $2 \%$ CHAPS, $0.5 \%$ IPG buffer and $18 \mathrm{mM}$ DTT. After passive rehydration for $12 \mathrm{~h}, \mathrm{IEF}$ was performed at a step voltage of $500 \mathrm{~V}$ for $1 \mathrm{~h}, 1000 \mathrm{~V}$ for $1 \mathrm{~h}$ and $8000 \mathrm{~V}$ for $5 \mathrm{~h}$. Following IEF separation, the gel strips were equilibrated for $2 \times 15 \mathrm{~min}$ in equilibration buffer containing $50 \mathrm{mM}$ Tris- $\mathrm{HCl}, \mathrm{pH} 8.8,6 \mathrm{M}$ urea, $2 \%$ SDS and $30 \%$ glycerol. About $1 \%$ DTT was added to the first equilibration buffer and in the second equilibration buffer, DTT was replaced with $4 \%$ iodoacetamide. The 2nd-dimensional electrophoresis was performed on $12.5 \%$ SDS-polyacrylamide gels with the SE 600 System (Amersham) at $30 \mathrm{~mA}$ per gel for $40 \mathrm{~min}$ and then at $60 \mathrm{~mA}$ until the bromophenol blue reached the end of the gel. Each sample was repeated three times. The analytical gels were stained with silver as previously described (Hochstrasser et al., 1988a, b) and the preparative gels were stained with coomassie blue.

Immunoblotting: Proteins from the same two gels were electrotransferred synchronously to two hybond-C nitrocellulose membranes (Amersham Biosciences) using the Trans Blot cell (Bio-Rad) for $90 \mathrm{~min}$ at $0.22 \mathrm{~A}$. Following the transfer, the membranes were stained with ponceau $\mathrm{S}$ solution (Ameresco) to check the transfer efficiency and then were incubated in blocking solution $(1 \% \mathrm{BSA}$ in $1 \times \mathrm{PBS})$ overnight at room temperature. The two membranes were subjected to 3 separate $15 \mathrm{~min}$ washes with $0.05 \%$-PBST after blocking. Then, one membrane was incubated at room temperature for $2 \mathrm{~h}$ in a pool of pre-treatment serum samples (diluted 1:100 in PBST) from ten randomly selected individuals as mentioned before. The other membrane was simultaneously treated in the same way with a pool of post-treatment serum samples (confirmed to be negative 20 weeks after $\mathrm{PZQ}$ treatment) from the same ten individuals as for pre-treatment (related demographics of these serum samples are in supplementary 4). After washing in PBST $(3 \times 15 \mathrm{~min})$, HRP conjugated goat anti-human IgG (1: 2000 in 0.05\% PBST, Sigma, USA) was 
added. The membrane was incubated at room temperature for $1 \mathrm{~h}$ and then washed 3 times in $0.05 \%$ PBST. The membrane was developed using $\mathrm{H}_{2} \mathrm{O}_{2}$ and 3,3'-Diaminobenzidine (DAB) in PBS. A pool of ten samples of normal human sera served as a control and was treated in the same way. The gel electrophoresis and Western blotting were repeated twice for all samples for confirmation.

Image analysis and mass spectrometry: Images from the immunoblot patterns were obtained by scanning with a Bio-Rad GS710 scanner and image analysis was accomplished using Image Master Software (Amersham) to match the reacted spots. Predicted matches were also visually verified. Protein spots on the coomassie blue stained gel that matched those on the Western blots were excised from the preparative gels and then destained with $100 \mathrm{mM} \mathrm{NH}_{4} \mathrm{HCO}_{3}$ in $30 \% \mathrm{ACN}$. After removing the destaining buffer, the gel pieces were lyophilized and rehydrated in $30 \mu \mathrm{L}$ of $50 \mathrm{mMNH}_{4} \mathrm{HCO}_{3}$ containing $50 \mathrm{ng}$ trypsin (sequencing grade; Promega, Madison, WI, USA). After overnight digestion at $37^{\circ} \mathrm{C}$, the peptides were extracted three times with $0.1 \% \mathrm{TFA}$ in $60 \% \mathrm{ACN}$. Extracts were pooled together and lyophilized. The resulting lyophilized tryptic peptides were kept at $-80^{\circ} \mathrm{C}$ until MS analysis. A protein-free gel piece was treated as above and used as a control to identify autoproteolysis products derived from trypsin.

MS and MS/MS spectra were obtained using the ABI 4800 Proteomics Analyzer MALDI-TOF/TOF (Applied Biosystems, Foster city) operating in a result dependent acquisition mode. Peptide mass maps were acquired in a positive ion reflector mode ( $20 \mathrm{kV}$ accelerating voltage) with 1000 laser shots per spectrum. Monoisotopic peak masses were automatically determined within the mass range $800-4000 \mathrm{Da}$ with a signal to noise ratio minimum set to 10 and a local noise window width of $250 \mathrm{~m} / \mathrm{z}$. Up to five of the most intense ions with a minimum signal to noise ratio of 50 were selected as precursors for MS/MS acquisition, excluding common trypsin autolysis peaks and matrix ion signals. In the MS/MS positive ion mode, spectra were averaged, collision energy was $2 \mathrm{kV}$ and the default calibration was set. Monoisotopic peak masses were automatically determined with a signal to noise ratio minimum set to 5 and a local noise window width of $250 \mathrm{~m} / \mathrm{z}$. The MS together with MS/MS spectra were searched against Schistosoma NCBI 2010-3-12 using the Software GPS Explorer 3.6 (Applied Biosystems) and MASCOT 2.1 (Matrix Science) with the following parameter settings: trypsin cleavage, one missed cleavage allowed, carbamidomethylation set as fixed modification, oxidation of methionine allowed as variable modification, peptide mass tolerance set to $100 \mathrm{ppm}$, fragment tolerance set to $\pm 0.3 \mathrm{Da}$ and the minimum ion score confidence interval for MS/MS data set to $95 \%$.

\section{RESULTS AND DISCUSSION}

2-DE: In this study, researchers carried out 2-DE analysis of the proteins of SEAs as described before. The total proteins from SEA were separated on 2-DE gels with a $\mathrm{pH}$ gradient from $\mathrm{pH} 3-10$ (Fig. 1). The 2-DE gels were stained with coomassie blue and $>300$ discrete spots were displayed. Most of them were located between $\mathrm{pH} 4$ and 8. Each sample was subjected to triplicate runs.

Western blot analysis: To investigate whether the components of S. japonicum SEA could be recognized by the pooled human serum samples collected before and after PZQ treatment, Western blot assays were performed on the basis of the $2 \mathrm{D}$ gels in the range $\mathrm{pH} 3-10$. Compared with the hybridized membrane of normal human sera (Fig. 2), researchers observed that $>80$ strong

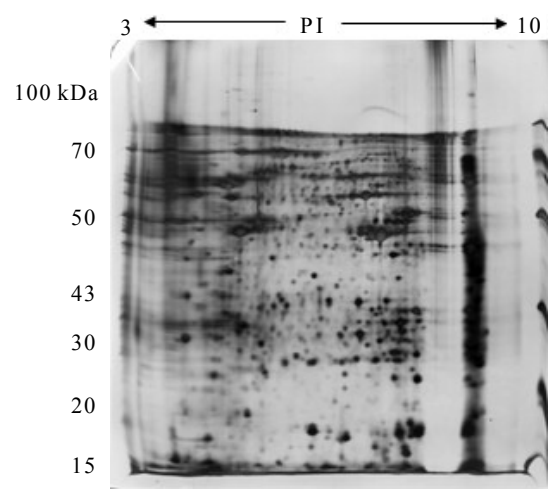

Fig. 1: 2-dimensional image of SEAs from S. japonicum. Coomassie blue stained gels with a range of $\mathrm{pH}$ 3-10. Molecular mass markers $(\mathrm{kDa})$ are given on the left

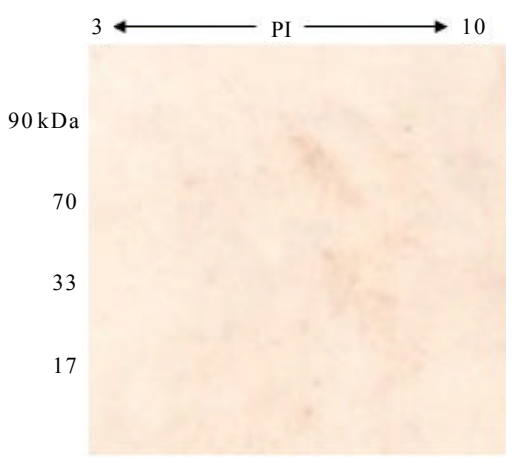

Fig. 2: Normal control for S. japonicum SEA. Molecular mass markers (in $\mathrm{kDa}$ ) are given on the left 
reactive spots displayed on the membranes hybridized with the pre treatment and post treatment human sera. There were differences between these two immunoblot patterns (Fig. 3A and B) while no significant immunoreactive spots were recognized by the normal human sera. Image analysis of $2 \mathrm{D}-\mathrm{WB}$ showed that a total of 84 protein spots were recognized in the pre and post treatment sera.

MS/MS analysis: After matching the immunoblots with their homologous coomassie blue stained gels of SEA with Image Mater 2D gel software (Amersham), researchers localized the spots that corresponded to the antigenic proteins in the $2 \mathrm{D}$ gels. In total, 84 matched spots were excised from the coomassie blue stained gels, digested by typsin and were further analyzed by LC/MS-MS (Fig. 4). The MS/MS raw data were used to search $S$. japonicum and $S$. mansoni protein public databases with the SEQUEST search program. Finally, 67 spots were successfully identified. The identified spots with their summary protein scores, isoelectric points (pI) and molecular mass $(\mathrm{kDa})$ are shown in Table 2. MS/MS analysis suggested that some different spots were derived from the same proteins. For example, spots $48,49,50,51$, 56 and 57 all referred to Glyceraldehyde 3-Phosphate Dehydrogenase (GAPDH) as these spots corresponded to the same proteins but with different NCBI accession numbers.

For the reason above, the 67 identified protein spots were grouped into 37 different proteins by molecular functions which included chaperone proteins, motor proteins, catabolic activity, binding proteins and others
(Table 3). Furthermore, 1 unknown protein and 3 hypothetical proteins without functional annotation are also shown in Table 3. The raw data of peptide mass flngerprints and secondary MS are shown in supplementary 1 and 2 . The peptide data (sequence, ion score and C. I. \%) are listed in the supplementary 3.

The effect of PZQ treatment on serological recognition: Comparative immunomic analysis of S. japonicum SEA recognized by the patient sera of pre and post treatment with $\mathrm{PZQ}$ was performed to investigate how chemotherapy altered responses to the antigens. The results suggested that treatment with $P Z Q$ mainly affected the intensity of serological recognition of $S$. japonicum SEA. The reactivity of most protein spots to sera was reduced by chemotherapy with $\mathrm{PZQ}$ after 20 weeks (Table 2). A total of 49 spots showed reduced reactivity. The most apparent spots included spots 25-27 (ATP synthase alpha subunit mitochondrial and 2 hypothetical proteins), 34-37 (branched chain amino acid aminotransferase, lactate dehydrogenase $\mathrm{A}$ and heterogeneous nuclear ribonucleoprotein A2 homolog 1) and 44-58 (actin, glyceraldehyde-3-phosphate dehydrogenase, troponin $\mathrm{T}$, major egg antigen $\mathrm{P} 40$, phosphatase $2 \mathrm{~A}$ inhibitor $\mathrm{I} 2 \mathrm{PP} 2 \mathrm{~A}$, tubulin beta 2 , ester hydrolase $\mathrm{C} 11$ orf 54 homolog and an unknown protein). However, only five spots $(1,3,17,18$ and 19) showed increases in the intensity of recognition after treatment. They were the dentin sialophosphoprotein precursor, $\mathrm{KH}$ type splicing regulatory protein and dihydropyrimidinase like 2.

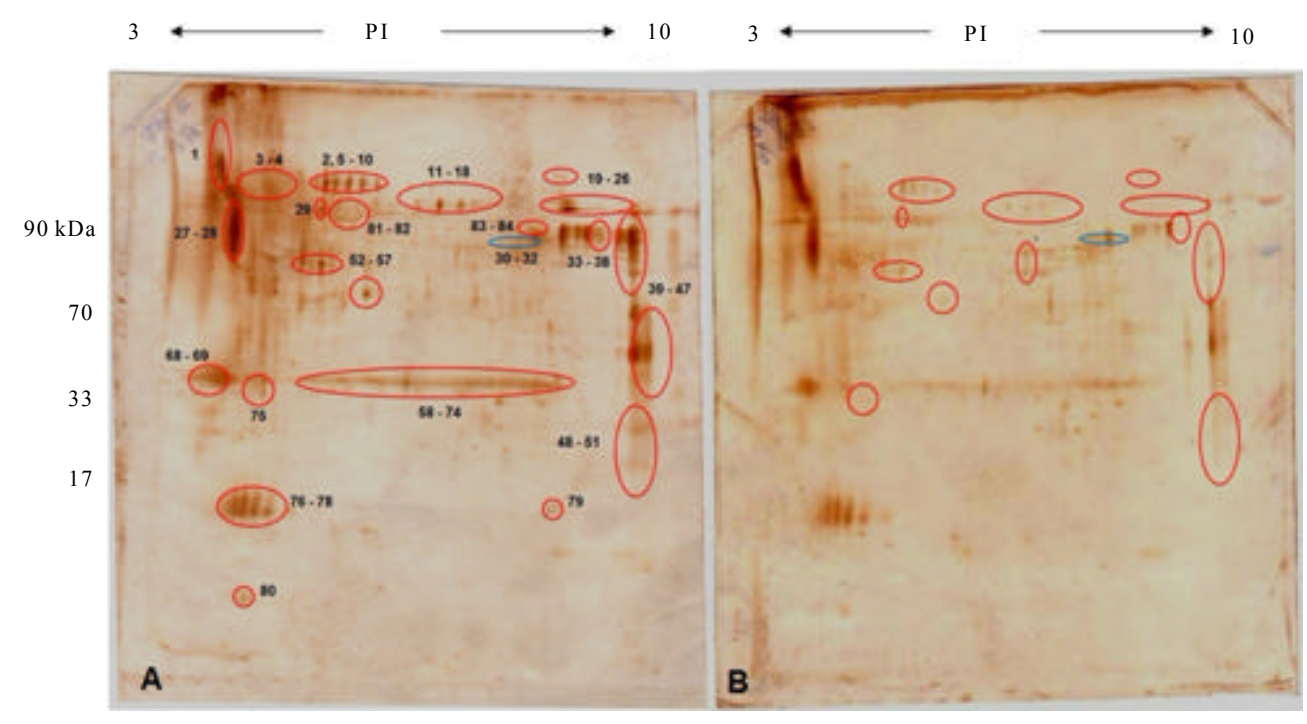

Fig. 3: Immunoblot patterns of S.japonicum SEAs recognized by serum samples. A: Spots reacting with pre treatment sera; B: Spots reacting with post treatment sera. Red circles represent the different areas between pre treatment and post treatment. Molecular mass markers $(\mathrm{kDa})$ are given on the left 


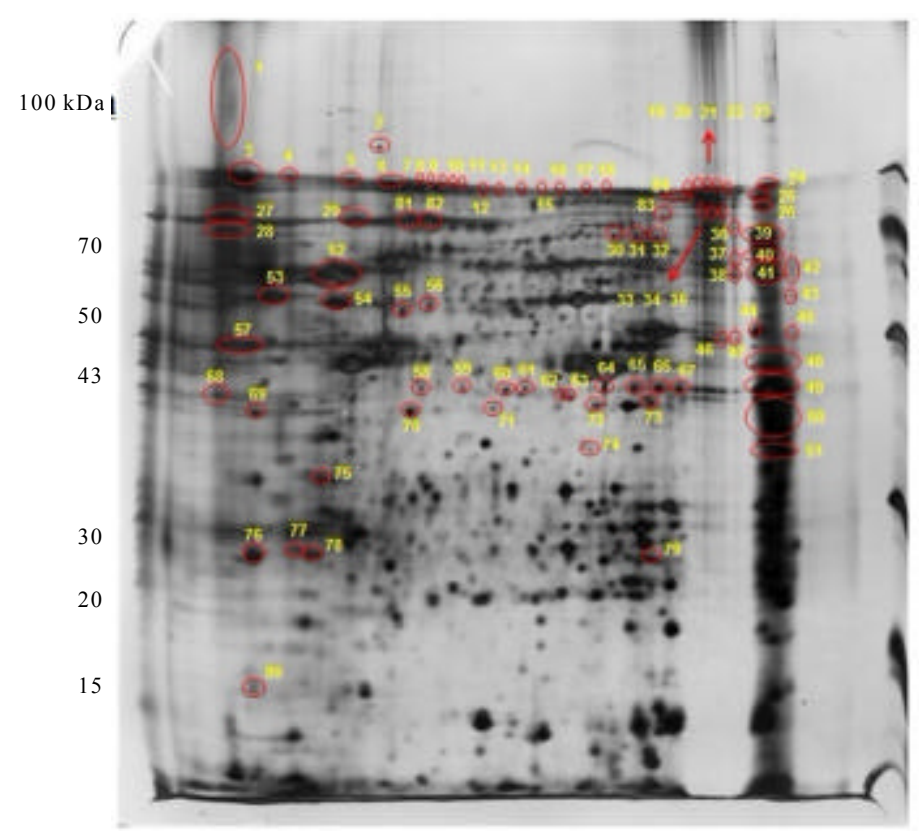

Fig. 4: Coomassie blue stained 2-D gel of S. japonicum SEAs showing spots matched to immunoblots. Molecular mass markers $(\mathrm{kDa})$ are given on the left

Table 2: Identities of $S$. joponicum SEA antigenic proteins recognized by serum samples

\begin{tabular}{|c|c|c|c|c|c|c|c|}
\hline Spot No. & Protein name & Species & NCBI Accession No. & Protein score & PI & Daltons & Trend \\
\hline 1 & Dentin sialophosphoprotein precursor & S. joponicum & gi|226485495 & 116 & 4.13 & 51762.9 & 1 \\
\hline 2 & Actin & S. monsoni & gi| 256079407 & 95 & 5.30 & 41704.8 & 1 \\
\hline 3 & Dentin sialophosphoprotein precursor & S. joponicum & gi|226480886 & 218 & 4.28 & 49969.7 & 1 \\
\hline 4 & Endoplasmin & S. mansoni & gi| 7673568 & 86 & 5.14 & 90445.1 & 1 \\
\hline 5 & Myosin heavy chain & S. mansoni & gi|238665121 & 83 & 5.70 & 222168.2 & 1 \\
\hline 6 & Rootletin (Ciliary rootlet coiled coil protein) & S. mansoni & gi| 238661382 & 71 & 5.92 & 233729.8 & 1 \\
\hline 7 & Heat shock protein 70 (HSP70)-4, putative & S. mansoni & gi| 238663484 & 60 & 5.43 & 94404.3 & 1 \\
\hline 8 & SJCHGC04997 protein & S. joponicum & gi|76155264 & 91 & 5.57 & 26838.0 & 1 \\
\hline 9 & Tubulin tyrosine ligase related & S. mansoni & gi|238667196 & 62 & 9.33 & 156758.0 & 1 \\
\hline 10 & Uracil DNA glycosylase, putative & S. mansoni & gi| 238663347 & 58 & 6.84 & 34927.8 & 1 \\
\hline 11 & Hypothetical protein & S. joponicum & gi|226468334 & 92 & 5.92 & 86311.6 & 1 \\
\hline 12 & Aconitate hydratase & S. mansoni & gi|238662901 & 129 & 8.51 & 85529.7 & 1 \\
\hline 13 & Arginine kinase & S. joponicum & gi|226481457 & 171 & 8.46 & 80133.0 & 1 \\
\hline 14 & Heat shock protein 70 (HSP70), putative & S. monsoni & gi| 238667420 & 431 & 5.36 & 68960.1 & - \\
\hline 15 & Phosphoenolpyruvate carboxykinase, putative & S. mansoni & gi|238657216 & 148 & 6.43 & 70345.2 & - \\
\hline 16 & HSP70 & S. joponicum & gi|2829289 & 713 & 5.12 & 71442.2 & 1 \\
\hline 17 & Putative KH-type splicing regulatory protein & S. joponicum & gi|257205944 & 247 & 6.73 & 54188.8 & 1 \\
\hline 18 & $\begin{array}{l}\text { KH-type splicing regulatory protein (FUSE } \\
\text { binding protein 2) }\end{array}$ & S. joponicum & gi|226477992 & 108 & 7.08 & 56457.9 & 1 \\
\hline 19 & Dihy dropyrimidinase-like 2 & S. joponicum & gi|226468250 & 128 & 7.54 & 63647.0 & 1 \\
\hline 20 & Heat shock protein 70 , putative & S. mansoni & gi| 238667420 & 62 & 5.36 & 68960.1 & - \\
\hline 21 & Heat shock protein 70 , putative & S. mansoni & gi|238667421 & 125 & 5.42 & 69785.6 & - \\
\hline 22 & Heat shock protein 70 , putative & S. joponicum & gi| 238667420 & 55 & 5.36 & 68960.1 & - \\
\hline 23 & Alpha tubulin, putative & S. mansoni & gi| 238665538 & 98 & 4.92 & 48380.7 & 1 \\
\hline 24 & $\begin{array}{l}\text { ATP synthase alpha subunit } \\
\text { mitochondrial, putative }\end{array}$ & S. mansoni & gi|238656902 & 104 & 9.16 & 59606.7 & 1 \\
\hline 25 & Hypothetical protein & S. joponicum & gi|226471882 & 108 & 8.67 & 34871.8 & 1 \\
\hline 26 & Hypothetical protein & S. joponicum & gi| 257216372 & 180 & 8.67 & 55850.4 & 1 \\
\hline 27 & $\begin{array}{l}\text { ATP synthase alpha subunit } \\
\text { mitochondrial, putative }\end{array}$ & S. monsoni & gi|238656902 & 207 & 9.16 & 59606.7 & $t$ \\
\hline 28 & Putative tubulin, beta, 2 & S. joponicum & gi|226467269 & 145 & 4.79 & 49802.9 & 1 \\
\hline 29 & Enolase & S. joponicum & gi 257123775 & 61 & 6.18 & 47221.0 & 1 \\
\hline 30 & Phosphogly cerate kinase 1 & S. joponicum & gi| 226475588 & 272 & 6.76 & 44252.8 & 1 \\
\hline 31 & Actin, putative & S. mansoni & gi|238661234 & 233 & 5.30 & 41706.8 & 1 \\
\hline 32 & Actin, putative & S. mansoni & gi| 238663895 & 159 & 5.30 & 35366.6 & $t$ \\
\hline
\end{tabular}


Table 2: Continue

\begin{tabular}{|c|c|c|c|c|c|c|c|}
\hline Spot No. & Protein name & Species & NCBI Accession No. & Protein score & PI & Daltons & Trend \\
\hline 33 & Actin, putative & S. japonicum & gi|238661234 & 230 & 5.30 & 41706.8 & 1 \\
\hline 34 & Branched-chain amino acid aminotransferase & S. mansoni & gi|238663016 & 145 & 8.56 & 42818.8 & 1 \\
\hline 35 & Lactate dehy drogenase $\mathrm{A}$ & S. japonicum & gi|226471614 & 275 & 8.45 & 36103.9 & 1 \\
\hline 36 & $\begin{array}{l}\text { Heterogeneous nuclear ribonucleoprotein } \\
\text { A2 homolog } 1\end{array}$ & S. japonicum & gi|226473314 & 163 & 8.63 & 33823.0 & t \\
\hline 37 & Hypothetical protein RNA-binding region RNP-1 & S. japonicum & gi|29841467 & 256 & 8.83 & 26766.6 & 1 \\
\hline 38 & Alpha tubulin, putative & S. mansoni & gi| 238665538 & 580 & 4.92 & 48380.7 & t \\
\hline 39 & Calreticulin & S. japonicum & gi|226473210 & 187 & 4.59 & 45832.3 & 1 \\
\hline 40 & $\begin{array}{l}\text { ATP synthase, } \mathrm{H}+\text { transporting, mitochondrial } \\
\text { F1 complex, beta polypeptide }\end{array}$ & S. japonicum & gi| 226487050 & 885 & 5.21 & 55762.9 & 1 \\
\hline 41 & Heat shock protein 60 & S. japonicum & gi|257215736 & 337 & 6.68 & 50571.6 & $t$ \\
\hline 42 & $26 \mathrm{~S}$ protease regulatory subunit $6 \mathrm{a}$, putative & S. japonicum & gi|238660820 & 333 & 5.61 & 41048.5 & 1 \\
\hline 43 & Actin & S. mansoni & gi|256079407 & 399 & 5.30 & 41704.8 & 1 \\
\hline 44 & Actin 5C & S. japonicum & gi|226472932 & 224 & 5.66 & 40099.1 & 1 \\
\hline 45 & Troponin $\mathrm{T}$ & S. japonicum & gi|226478018 & 73 & 6.04 & 38401.4 & 1 \\
\hline 46 & Major egg antigen (P40) & S. japonicum & gi|226475110 & 266 & 6.79 & 28337.1 & t \\
\hline 47 & Major egg antigen (P40) & S. japonic um & gi|226477156 & 290 & 6.28 & 39155.4 & 1 \\
\hline 48 & Glyceraldehyde 3-phosphate dehydrogenase & S. japonicum & gi|226477756 & 141 & 6.76 & 31964.2 & 1 \\
\hline 49 & Glyceraldehyde 3-phosphate dehydrogenase & S. japonicum & gi|226477740 & 204 & 7.60 & 31991.2 & 1 \\
\hline 50 & Glyceraldehyde 3-phosphate dehydrogenase & S. japonicum & gi|1916689 & 233 & 8.11 & 36551.6 & 1 \\
\hline 51 & Glyceraldehyde 3-phosphate dehydrogenase & S. japonicum & gi|257209371 & 61 & 7.59 & 33347.0 & 1 \\
\hline 52 & Glyceraldehyde 3-phosphate dehydrogenase & S. japonicum & gi|226477756 & 61 & 7.60 & 31964.2 & 1 \\
\hline 53 & Phosphatase $2 \mathrm{~A}$ inhibitor I2PP $2 \mathrm{~A}$ & S. japonicum & gi|226477576 & 142 & 5.05 & 18716.3 & 1 \\
\hline 54 & Putative tubulin, beta, 2 & S. japonicum & gi|226471718 & 394 & 5.29 & 37017.1 & 1 \\
\hline 55 & Major egg antigen (p40) & S. japonicum & gi|226477150 & 286 & 6.40 & 39214.5 & 1 \\
\hline 56 & Glyceraldehyde 3-phosphate dehydrogenase & S. japonicum & gi|226477756 & 141 & 6.76 & 31964.2 & 1 \\
\hline 57 & Glyceraldehyde 3-phosphate dehydrogenase & S. japonicum & gi|226477740 & 204 & 7.60 & 31991.2 & 1 \\
\hline 58 & Ester hy drolase C11 orf 54 homolog & S. japonicum & gi|226481407 & 174 & 7.19 & 21866.0 & 1 \\
\hline 59 & Heat shock protein HSP60, putative & S. mansoni & gi|238657488 & 91 & 6.35 & 60932.7 & - \\
\hline 60 & Conserved hypothetical protein & S. japonicum & gi|226483787 & 105 & 4.36 & 20908.8 & - \\
\hline 61 & Putative tubulin, beta, 2 & S. japonicum & gi|226471718 & 115 & 5.29 & 37017.1 & - \\
\hline 62 & $\begin{array}{l}\text { Myosin regulatory light chain 2, smooth } \\
\text { muscle minor isoform (G1) }\end{array}$ & S. japonicum & gi|226469350 & 123 & 5.23 & 20742.2 & - \\
\hline 63 & Hypothetical protein & S. japonicum & gi|226473034 & 291 & 8.56 & 24987.8 & - \\
\hline 64 & $20 \mathrm{kDa}$ calcium-binding protein (Antigen SM20) & S. japonicum & gi|226489228 & 122 & 4.34 & 17868.8 & t \\
\hline 65 & Heat shock protein 70 , putative & S. mansoni & gi| 238667420 & 332 & 5.36 & 68960.1 & - \\
\hline 66 & Heat shock protein 70 , putative & S. mansoni & gi|238667421 & 338 & 5.42 & 69785.6 & - \\
\hline 67 & Putative UDP-galactose-4-epimerase & S. japonicum & gi 226487124 & 63 & 7.55 & 72326.7 & - \\
\hline
\end{tabular}

During the complex life stages of schistosomes, the egg is the key agent responsible for both the transmission and pathogenesis of schistosomiasis. Immunopathology in schistosomiasis is due to granulomatous inflammation around parasite eggs in the host liver and intestines which may result in scarring, portal hypertension, haemorrhage and death. The treatment and control of schistosomiasis relies virtually on a single drug, $\mathrm{PZQ}$. However, chemotherapy with PZQ causes a series of problems for serological diagnosis of schistosomiasis (Pearce and MacDonald, 2002; Mutapi et al., 1998; Martins-Leite et al., 2008). Characterization of the SEA immunome may provide clues for potential diagnosis markers and for broader understanding of the egg's interaction with the mammalian host. In this study, a combination of 2-dimensional electrophoresis and Western blotting was employed to profile $S$. japonicum SEAs with pooled human serum of pre and post treatment by PZQ.
A total of 84 proteins spots recognized by human infection sera were subjected to MS analysis and 37 different characterized proteins were successfully identified, four of which have no functional annotation. Moreover of these, the reactivity of 49 protein spots (30 characterized proteins) to sera was reduced after 20 weeks by chemotherapy with PZQ whereas only five spots (3 different characterized proteins) showed increases in the intensity of recognition after treatment.

Of the 37 different immunogenic proteins identified, proteins related to catabolic activity were the most abundant, forming the largest functional category in SEA. This is probably because eggs are rich in carbohydrates and polysaccharide antigens and tend to stimulate an IgM response (Woolhouse and Hagan, 1999). Consequently, numerous enzymes might be involved in PZQ chemotherapy. For instance, one of the most abundant proteins with catabolic activity, glyceraldehyde-3 phosphate dehydrogenase (GAPDH; spots 48, 49, 50, 51, 


\begin{tabular}{|c|c|}
\hline Function category & Protein identity \\
\hline \multirow[t]{3}{*}{ Chaperone } & Heat shock protein 60 \\
\hline & Heat shock protein 70 \\
\hline & Major egg antigen (P40) \\
\hline \multirow[t]{5}{*}{ Motor protein } & Actin \\
\hline & Tubulin \\
\hline & Troponin $\mathrm{T}$ \\
\hline & Myosin heavy chain \\
\hline & $\begin{array}{l}\text { Myosin regulatory light chain } 2 \text {, smooth muscle } \\
\text { minor isoform (G1) }\end{array}$ \\
\hline \multirow[t]{15}{*}{ Catabolic activity } & Enolase \\
\hline & Aconitate hy dratase \\
\hline & Arginine kinase \\
\hline & Phosphoglycerate kinase 1 \\
\hline & Phosphoenolpyruvate carboxykinase, putative \\
\hline & Glyceraldehyde 3-phosphate dehydrogenase \\
\hline & Dihydropyrimidinase-like 2 \\
\hline & ATP synthase alpha subunit mitochondrial, putative \\
\hline & Lactate dehy drogenase $\mathrm{A}$ \\
\hline & Uracil-DNA glycosylase, putative \\
\hline & Branched-chain amino acid aminotransferase \\
\hline & Tubulin tyrosine ligase related \\
\hline & Ester hy drolase C11 orf 54 homolog \\
\hline & Endoplasmin \\
\hline & Putative UDP-galactose-4-epimerase \\
\hline \multirow[t]{5}{*}{ Bingding } & Calreticulin \\
\hline & $20 \mathrm{kDa}$ calcium-binding protein (Antigen SM20) \\
\hline & ATP synthase, $\mathrm{H}+$ transporting, mitochondrial $\mathrm{F} 1$ \\
\hline & complex, beta polypeptide \\
\hline & $\begin{array}{l}\text { KH-type splicing regulatory protein (FUSE } \\
\text { binding protein 2) }\end{array}$ \\
\hline \multirow[t]{8}{*}{ Other } & Dentin sialophosphoprotein precursor \\
\hline & Rootletin (Ciliary rootlet coiled-coil protein), putative \\
\hline & Phosphatase $2 \mathrm{~A}$ inhibitor I2PP $2 \mathrm{~A}$ \\
\hline & $26 \mathrm{~S}$ protease regulatory subunit $6 \mathrm{a}$, putative \\
\hline & Heterogeneous nuclear ribonucleoprotein A2 \\
\hline & SJCHGC04997 protein \\
\hline & SJCHGC 03722 protein \\
\hline & Hypotherical protein (spots 11, 25 and 26) \\
\hline
\end{tabular}

56 and 57) which was considered as a putative vaccine candidate against $S$. japonicum showed a correlation of antibody titer with resistance to reinfection (Waine et al., 1993; Argiro et al., 2000). Its abundant expression across developmental stages of S. mansoni (Curwen et al., 2004) indicated that GAPDH is associated with the growth and development of schistosomes. Furthermore, MS/MS analysis identified a glycolytic and gluconeogenic enzyme i.e., enolase which is also found in excretory/secretory proteins of schistosome adult worms with high expression (Liu et al., 2009a). Many studies have shown that enolase can be found on the surface of several eukaryotes and prokaryotes including schistosomes in which it acts as a plasminogen binding receptor (Ramajo-Hernandez et al., 2007; Seweryn et al., 2007). It is well known that energy acquisition by schistosomes relies mainly on the glycolytic pathway and enolase is a key enzyme in this pathway. Enolase exhibited only poor protection against S. japonicum schistosomulum infection (Yang et al., 2010) but assessment of its potential as a diagnostic target has yet to be reported.
The chaperone group such as Heat Shock Proteins (HSP) was another abundantly expressed group of immunogenic proteins in S. japonicum SEA. Both HSP60 and HSP70 were identified in this investigation. Serological evaluation of HSP60 demonstrated that the sensitivity of this antigen was $>90 \%$ with ELISA. HSP 70 is considered the predominant HSP family and plays a key regulatory role in parasite development and pathogenesis. A DNA vaccine of SjGST combined with SjMLP/HSP70 could induce a $31.31 \%$ reduction of the worm burden and a $58.59 \%$ reduction of the egg burden in the intestinal tissue of immunized mice ( $\mathrm{He}$ et al., 2010). Another typical immunogen chaperone protein is a major egg antigen, P40. The pathogenesis of $S$. mansoni infection depends largely on host T-cell mediated immune responses such as the granulomatous response to tissue deposited eggs and subsequent fibrosis (Pearce and MacDonald, 2002). The major egg antigens have a valuable role in desensitizing the CD4+ Th cells that mediate granuloma formation which may prevent or ameliorate clinical signs of schistosomiasis (Abouel-Nour et al., 2006). There were significant differences after Smp40 stimulation in IL-5 and 10 levels compared with the control group. There is no information available on the changes in reactivity of this antigen following chemotherapy before this study. Among the recognized proteins, motor and binding proteins are the other two groups of immunogens. The former group might be involved in the formation and development of eggs. It is generally believed that $\mathrm{Ca}^{2+}$ channels are the molecular targets of $\mathrm{PZQ}$ action (Greenberg, 2005). Interestingly, the latter group comprises components of $\mathrm{Ca}^{2+}$ ion signaling pathways such as calreticulin and calcium binding protein. In addition, these immunogens also include some S. japonicum egg-specific proteins such as troponin $\mathrm{T}$ and their biological characterization needs to be further explored.

Researchers found that some relatively heavily stained gel spots failed to be recognized by serum samples which is consistent with the results for S. haematobium Soluble Worm Antigens (SWA) (Mutapi et al., 2005). Mature schistosomes have evolved highly with effective mechanisms for evading the consequences of the cellular and humoral immune responses that they provoke (Pearce and Sher, 1987; Pearce and MacDonald, 2002). Perhaps these unrecognized SEA components also play a vital role in host immune evasion or modulation and hence deserve attention and further evaluation.

Comparative analysis on the serological recognition of SEA revealed that chemotherapy with $P Z Q$ suppressed the reactivity of most of SEA spots to human sera. On the 
contrary, PZQ could increase the intensity of recognition for some SWA spots after treatment (Mutapi et al., 2005). As a single drug for the treatment of schistosomiasis, it is well established that $\mathrm{PZQ}$ exerts different actions on mature or immature adult worms and eggs: PZQ kills adult worms (Andrews, 1985) and mature eggs (Richards et al., 1989) but does not significantly affect immature worms (Sabah et al., 1986). PZQ can damage the tegument of adult worms and exposes more parasite antigens to the host immune system compared with natural infection (Mutapi, 2001). The changes in serological recognition of SEA might be directly ascribed to the reduction of egg burden because of $\mathrm{PZQ}$ induced worm death. Moreover, although PZQ has no effect on $S$.japonicum eggs trapped in tissues, previous research showed that $\mathrm{PZQ}$ can restrain schistosomal ovum granulomas ( $\mathrm{Xu}$ et al., 2007) Therefore, researchers presume that the decreases in serological recognition of SEA were probably related to the change of ovum granuloma after treatment. $P Z Q$ treatment resulted in marked changes in S. haematobium specific antibody responses directed against SEA which persisted from 18 weeks to at least 36 weeks following chemotherapy (Mutapi et al., 1998). This reminds us that extension of the sera samples collection time after chemotherapy may be able to reflect the dynamic changes of the immunogenic antigens researchers identified.

It is well known that antibodies of high affinity are more efficient than lower affinity antibodies in a wide range of biological reactions. Antibodies are traditionally considered to engage antigens in a mono specific bivalent manner which enhances apparent affinity by decreasing the rate of dissociation from the ligand. Heteroligation could improve antibody affinity when homotypic bivalent binding was not possible during the immune response to human immunodeficiency virus (Mouquet et al., 2010). Patients who developed AIDS either lost or failed to develop high affinity antibodies early in the infection. The antibody affinity values declined with time in some AIDS patients who remained asymptomatic (Chargelegue et al., 1995). Chemotherapy with $P Z Q$ caused changes in the amount of antigens directly into the bloodstream, thus stimulating different antigen presenting cells which resulted in different interactions between the antigens and the immune system when compared with natural infection (Mutapi, 2001). During this process, the alteration of SEA reactivity to sera after treatment may be associated with the change in the affinity of specific antibodies against SEA.

\section{CONCLUSION}

The present study suggested that chemotherapy with $\mathrm{PZQ}$ mainly affects the intensity of serological recognition of $S$. japonicum SEA. The immunomic proteins that were identified may facilitate a better understanding of the egg-induced pathogenesis of schistosomiasis and host-parasite interplay and may provide potential targets for the diagnosis and evaluation of treatment for the disease as well.

\section{ACKNOWLEDGEMENTS}

Projects supports were provided by grants from The National High Tech Research and Development Program of China (863) (Grant No. 2007AA02Z153), The National Science and Technology Key Project on Major Infectious Diseases such as HIV/AIDS, Viral Hepatitis Prevention and Treatment (Grant No. 2009ZX10004-302, 2008ZX10004-011). Researchers are grateful to Mrs. Ying $\mathrm{Wu}$ for the maintenance of $S$. japonicum life cycle in laboratory. The thanks are also given to Shanghai Applied Protein Technology Co. Ltd., for protein identification. C. Ju and B. Xu contributed equally to this work. W. Hu is another corresponding author: National Institute of Parasitic Diseases, Chinese Center for Disease Control and Prevention, 200025 Shanghai, P.R. China.

\section{REFERENCES}

Abdulla, M.H., K.C. Lim, M. Sajid, J.H. McKerrow and C.R. Caffrey, 2007. Schistosomiasis mansoni: Novel chemotherapy using a cysteine protease inhibitor. PLoS Med., Vol. 4. 10.1371/journal.pmed.0040014.

Abouel-Nour, M.F., M. Lotfy, A.M. Attallah and B.L. Doughty, 2006. Schistosoma mansoni major egg antigen Smp40: molecular modeling and potential immunoreactivity for anti-pathology vaccine development. Mem. Inst. Oswaldo Cruz, $101: 365-372$

Andrews, P., 1985. Praziquantel: Mechanisms of antischistosomal activity. Pharmacol. Ther., 29: 129-156.

Argiro, L.L., S.S. Kohlstadt, S.S. Henri, H.H. Dessein and V.V. Matabiau et al., 2000. Identification of a candidate vaccine peptide on the $37 \mathrm{kDa}$ Schistosoma mansoni GAPDH. Vaccine, 18: 2039-2048.

Berriman, M., B.J. Haas, P.T. LoVerde, R.A. Wilson and G.P. Dillon et al., 2009. The genome of the blood fluke Schistosoma mansoni. Nature, 460: 352-358.

Boros, D.L. and K.S. Warren, 1970. Delayed hypersensitivity granuloma formation and dermal reaction induced and elicited by a soluble factor isolated from Schistosoma mansoni eggs. J. Exp. Med., 132: 488-507.

Cass, C.L., J.R. Johnson, L.L. Califf, T. Xu and H.J. Hernandez et al., 2007. Proteomic analysis of Schistosoma mansoni egg secretions Mol. Biochem. Parasitol., 155: 84-93. 
Chargelegue, D., C.M. Stanley, C.M. O'Toole, B.T. Colvin and M.W. Steward, 1995. The affinity of IgG antibodies to gag p24 and p17 in HIV-1-infected patients correlates with disease progression. Clin. Exp. Immunol., 99: 175-181.

Chitsulo, L., D. Engels, A. Montresor and L. Savioli, 2000. The global status of schistosomiasis and its control. Acta Trop., 77: 41-51.

Cioli, D., 2000. Praziquantel: Is there real resistance and are there alternatives? Curr. Opin. Infect. Dis., 13: $659-663$.

Curwen, R.S., P.D. Ashton, D.A. Johnston and R.A. Wilson, 2004. The Schistosoma mansoni soluble proteome: A comparison across four lifecycle stages. Mol. Biochem. Parasitol., 138: 57-66.

Dalton, J.P., S.R. Day, A.C. Drew and P.J. Brindley, 1997. A method for the isolation of schistosome eggs and miracidia free of contaminating host tissues. Parasitology, 115: 29-32.

Doenhoff, M. and P.L. Mattoccia, 2006. Praziquantel for the treatment of schistosomiasis: Its use for control in areas with endemic disease and prospects for drug resistance. Exp. Rev. Ant. Infect. Ther., 4: $199-210$.

Doenhoff, M.J., J.R. Kusel, G.C. Coles and D. Cioli, 2002. Resistance of Schistosoma mansoni to praziquantel: Is there a problem? Trans. R. Soc. Trop. Med. Hyg., 96: 465-469.

Driguez, P., D.L. Doolan, A. Loukas, P.L. Felgner and D.P. McManus, 2010. Schistosomiasis vaccine discovery using immunomics. Parasites Vectors, Vol. 3. 10.1186/1756-3305-3-4.

Greenberg, R.M., 2005. Are $\mathrm{Ca}^{2+}$ channels targets of praziquantel action? Int. J. Parasitol., 35: 1-9.

He, S., L. Yang, Z. Lv, W. Hu and J. Cao et al., 2010. Molecular and functional characterization of a mortalin-like protein from Schistosoma japonicum (SjMLP/hsp70) as a member of the HSP70 family. Parasitol. Res., 107: 955-966.

Hochstrasser, D.F., A. Patchornik and C.R. Merril, 1988a. Development of polyacrylamide gels that improve the separation of proteins and their detection by silver staining. Anal. Biochem., 173: 412-423.

Hochstrasser, D.F., M.G. Harrington, A.C. Hochstrasser, M.J. Miller and C.R. Merril, 1988b. Methods for increasing the resolution of two-dimensional protein electrophoresis. Anal. Biochem., 173: 424-435.

Hotez, P.J., P.J. Brindley, J.M. Bethony, C.H. King, E.J. Pearce and J. Jacobson, 2008. Helminth infections: The great neglected tropical diseases. J. Clin. Invest., 118: 1311-1321.
Ju, C., Z. Feng, P.J. Brindley, D.P. McManus, Z. Han, J.X. Peng and W. Hu, 2010. Our wormy world genomics, proteomics and transcriptomics in East and Southeast Asia. Adv. Parasitol., 73: 327-371.

Kassim, O. and D.E. Gibertson, 1976. Hatching of Schistosoma mansoni eggs and observations on motility of miracidia. J. Parasitol., 62: 715-720.

Katz, N., A. Chave and J. Pellegrino, 1972. A simple device for quantitative stool thicks mear technique in Schistosoma mansoni. Revista do Instituto de Medicina Tropical de Sao Paulo, 14: 397-400.

Keiser, J., J. Chollet, S.H. Xiao, J.Y. Mei, P.Y. Jiao, J. Utzinger and M. Tanner, 2009. Mefloquine: An aminoalcohol with promising antischistosomal properties in mice. PLoS Neglectrd Trop. Dis., Vol. 3. 10.1371/journal.pntd.0000350

Liu, F., S.J. Cui, W. Hu, Z. Feng, Z.Q. Wang and Z.G. Han, 2009a. Excretory/secretory proteome of the adult developmental stage of human blood fluke, Schistosoma japonicum. Mol. Cell Proteomics, 8: 1236-1251.

Liu, F., Y. Zhou, Z.Q. Wang, G. Lu and H. Zheng et al., 2009b. The Schistosoma japonicum genome reveals features of host-parasite interplay. Nature, 460: 345-351.

Martins-Leite, P., G. Gazzinelli, L.F. Alves-Oliveira, A. Gazzinelli and L.C.C. Malaquias et al., 2008. Effect of chemotherapy with praziquantel on the production of cytokines and morbidity associated with schistosomiasis mansoni. Antimicrob. Agents Chemother., 52: 2780-2786.

Mathieson, W. and R.A. Wilson, 2010. A comparative proteomic study of the undeveloped and developed Schistosoma mansoni egg and its contents: The miracidium, hatch fluid and secretions. Int. J. Parasitol., 40: 617-628.

Moore, D.V. and J.H. Sandground, 1956. The relative egg producing capacity of Schistosoma mansoni and Schistosoma japonicum. Am. J. Trop. Med. Hyg., 5: $831-840$.

Mouquet, H., J.F. Scheid, M.J. Zoller, M. Krogsgaard and R.G. Ott et al., 2010. Polyreactivity increases the apparent affinity of anti-HIV antibodies by heteroligation. Nature, 467: 591-595.

Mutapi, F., 2001. Heterogeneities in anti-schistosome humoral responses following chemotherapy. Trends Parasitol., 17: 518-524.

Mutapi, F., P.D. Ndhlovu, P. Hagan and M.E. Woolhouse, 1998. Changes in specific anti-egg antibody levels following treatment with praziquantel for Schistosoma haematobium infection in children. Parasite Immunol., 20: 595-600. 
Mutapi, F., R. Burchmore, T. Mduluza, A. Foucher and Y. Harcus et al., 2005. Praziquantel treatment of individuals exposed to Schistosoma haematobium enhances serological recognition of defined parasite antigens. J. Infect. Dis., 192: 1108-1118.

Pearce, E.J. and A. Sher, 1987. Mechanisms of immune evasion in schistosomiasis. Contrib. Microbiol. Immunol., 8: 219-232.

Pearce, E.J. and A.S. MacDonald, 2002. The immunobiology of schistosomiasis. Nat. Rev. Immunol., 2: 499-511.

Perez-Sanchez, R., A. Ramajo-Hernandez, V. RamajoMartin and A. Oleaga, 2006. Proteomic analysis of the tegument and excretory-secretory products of adult Schistosoma bovis worms. Proteomics, 6: S226-S236.

Ramajo-Hernandez, A., R. Perez-Sanchez, V. RamajoMartin and A. Oleaga, 2007. Schistosoma bovis: Plasminogen binding in adults and the identification of plasminogen-binding proteins from the worm tegument. Exp. Parasitol., 115: 83-91.

Renganathan, E. and D. Cioli, 1998. An international initiative on praziquantel use. Parasitol. Today, 14: $390-401$.

Richards, Jr., F., J. Sullivan, E. Ruiz-Tiben, M. Eberhard and H. Bishop, 1989. Effect of praziquantel on the eggs of Schistosoma mansoni, with a note on the implications for managing central nervous system schistosomiasis. Ann. Trop. Med. Parasitol., 83: $465-472$.

Sabah, A.A., C. Fletecher, G. Webbe and M.J. Doenhoff, 1986. Schistosoma mansoni: Chemotherapy of infections of different ages. Exp. Parasitol., 61: 294-303.

Sayed, A.A., A. Simeonov, C.J. Thomas, J. Inglese, C.P. Austin and D.L. Williams, 2008. Identification of oxadiazoles as new drug leads for the control of schistosomiasis. Nat. Med., 14: 407-412.

Seubert, J., R. Pohlke and F. Loebich, 1977. Synthesis and properties of praziquantel, a novel broad spectrum anthelmintic with excellent activity against schistosomes and cestodes. Cell. Mol. Life Sci., 33: $1036-1037$.
Seweryn, E., J. Pietkiewicz, A. Szamborska and A. Gamian, 2007. Enolase on the surface of prockaryotic and eukaryotic cells is a receptor for human plasminogen. Postepy. Hig. Med. Dosw., 61: 672-682.

Waine, G.J., M. Becker, W. Yang, B. Kalinna and D.P. McManus, 1993. Cloning, molecular characterization and functional activity of Schistosoma japonicum glyceraldehyde-3-phosphate dehydrogenase, a putative vaccine candidate against schistosomiasis japonica. Infect. Immunity, 61: 4716-4723.

Wang, L., J. Utzinger and X.N. Zhou, 2008. Schistosomiasis control: Experiences and lessons from China. Lancet, 372: 1793-1795.

Wilson, R.A., R.S. Curwen, S. Braschi, S.L. Hall, P.S. Coulson and P.D. Ashton, 2004. From genomes to vaccines via the proteome. Mem. Inst. Oswaldo Cruz, 99: 45-50.

Woolhouse, M.E. and P. Hagan, 1999. Seeking the ghost of worms past. Nat. Med., 5: 1225-1227.

Xiao, S.H., B.A. Catto and L.T. Webster Jr., 1985. Effects of praziquantel on different developmental stages of Schistosoma mansoni in vitro and in vivo. J. Infect. Dis., 151: 1130-1137.

Xiao, S.H., W.J. Yue, Y.Q. Yang and J.Q. You, 1987. Susceptibility of Schistosoma japonicum to different developmental stages to praziquantel. Chin. Med. J., 100: 759-768.

Xu, Y.L., Y.X. Huang, C.X. Yu, Y.S. Liang and Y.Y. $\mathrm{Wu}$ et al., 2007. Effect of praziquantel on schistosomal ovum granuloma and hepatic fibrosis I: Effect of praziquantel on schistosomal ovum granuloma in the lung of mice. Chin. J. Schisto Control, 19: 357-359.

Yang, J., C. Qiu, Y. Xia, L. Yao and Z. Fu et al., 2010. Molecular cloning and functional characterization of Schistosoma japonicum enolase which is highly expressed at the schistosomulum stage. Parasitol. Res., 107: 667-677.

Zhu, Y.C., 2005. Immunodiagnosis and its role in schistosomiasis control in China: A review. Acta Trop., 96: 130-136. 